Historic, Archive Document

Do not assume content reflects current scientific knowledge, policies, or practices. 



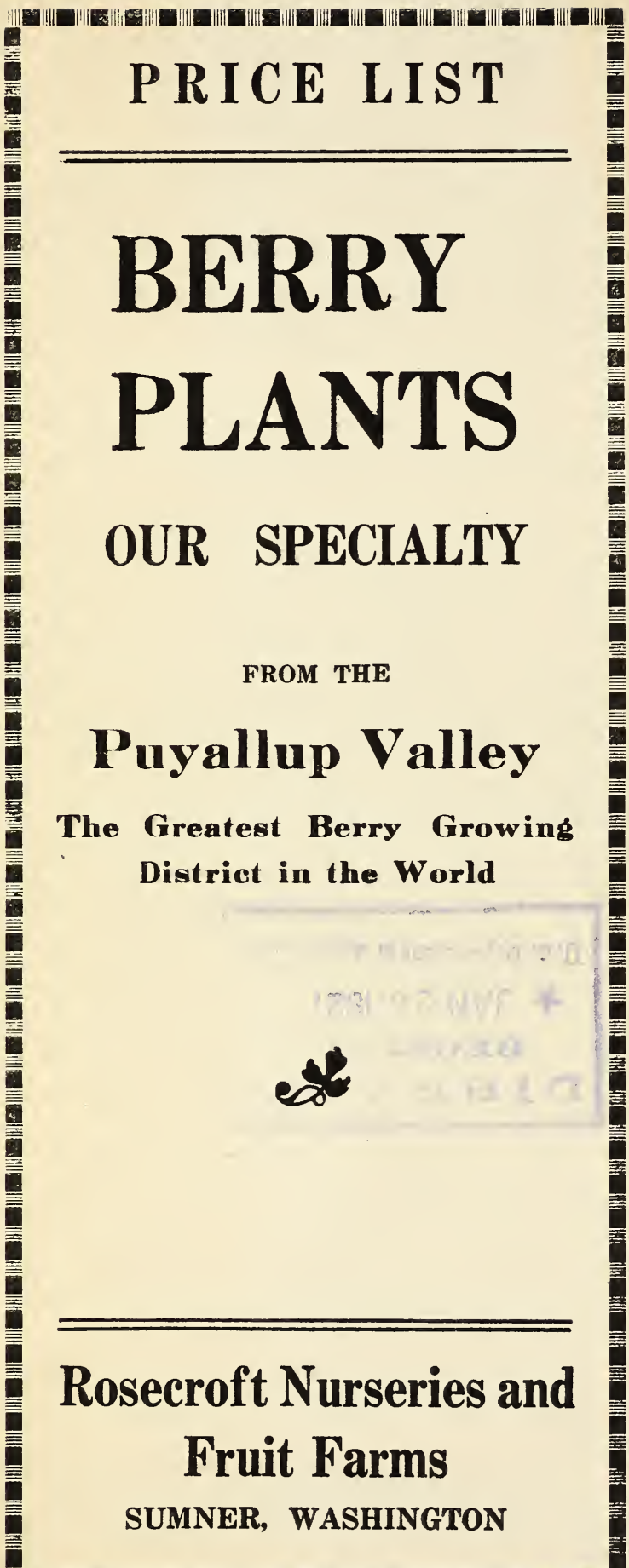

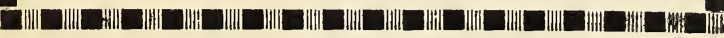




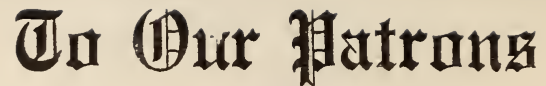

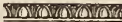

Our nurseries are located on paved highway about one mile southeast of Sumner postoffice. We make a specialty of small fruits as well as plants.

As we grow plants in very large quantities for other nurseries and for large planting, we can make especially attractive prices to those who are placing large orders.

We would advise ordering early, the earlier the better, as orders are filled in rotation as received; and the earliest selections are often the best stock.

SUBSTITUTING. In unusual cases we sometimes find it necessary to put in another kind in the place of the one ordered. This is due to our running short of the variety or inability to dig them at proper time. There are many varieties practically the same or that closely resemble each other in appearance and in value. 'In such cases we always endeavor to select varieties of equal or superior value to those ordered. If you do not wish us to exercise this method please mark your order sheet plainly "No substitution." We endeavor to give good value to all customers and use every precaution to send out only first-class stock and true to name. If any of our plants are found to be otherwise we will cheerfully replace them free of charge or refund the purchase price, but it is mutually understood between ourselves and our patrons that we are not liable for damages more than the original price paid for goods. We cannot guarantee all plants to live because it is impossible for us to know the conditions of planting and care they receive.

SEND CASH WITH ORDER, Check, postal order, express money order or stamps in small denomination, and if plants are to be sent by parcel post, include postage with order. If preferred, shipment may be made by parcel post or express C.O.D., that is, all the charges are to be paid by the customer when plants are received.

TERMS: 50 at 100 rate; 500, at 1000 rate.

The prices in this list do not include transportation.

It is difficult to furnish our customers with exact charges on packages by parcel post. Plants of all kinds vary in weight at different seasons of the year. 


\section{Rosecroft Nurseries}

\section{Sumner, Washington}

\section{ASPARAGUS}

$\begin{array}{llllr}\text { Palmetto ........... } & .20 & 1.00 & 7.50 \\ \text { Conover's Colossal .... } & .20 & 1.00 & 7.50 \\ \text { Mammoth White . . . . } & .20 & 1.00 & 7.50 \\ \text { 2-year Asparagus .... } & .25 & 1.25 & 10.00\end{array}$

Mad. Testout .................. each .75

Climbing Testout ................." " .75

Dorothy Perkins ................ " $\quad .50$

Climbing Hermosa ............... " $\quad .50$

Frau Karl Druschki ................ " $\quad .75$

Gen. Jack ................... "

Gen. McArthur ................ " $\quad .75$

Yellow Rambler ................... " $\quad .75$

K. A. Victoria .................. " $\quad .75$

La France .................. " 75

Mrs. A. R. Waddell ............. " $\quad .75$

Ophelia .................... " $\quad .75$

Lady Hillington ................. "

Hugh Dixon ................... " .75

W. A. Richardson ................. " $\quad .75$

Al. Bruner .................... "

Crimson Rambler ............... $\quad .5 \mathrm{c}$

\section{CHERRIES}

Bing ...............2 years.. each 1.00

Royal Ann .............2 years.. " 1.00

Sour Cherries ........... 2 years.. " 1.00

APPLES

Yellow Transparent .......2 2 years.. each .75

hing ................ 2 years.." 1.00

Winter Banana ...........2 years.. " 1.00

Delicious ..............2 2 years.. " 1.00

Wagner $\ldots \ldots \ldots \ldots \ldots \ldots \ldots 2$ years.. " 1.00

Gravenstein ..........2 2 years.. " 1.00

PEACHES

Early Crawford ................ each 80

Elberta ................... " 80

PEARS

Bartlett .................. each .75

Late Pears ................. " 75

PLUMS AND PRUNES

Erench Prunes ................. each .75

Silver Prunes .................. " $\quad .75$

Italian Prunes $\ldots \ldots \ldots \ldots \ldots \ldots \ldots \ldots \ldots, \quad .50$

Damson Plum ................... " $\quad .75$

Bradshaw Plum ................ " $\quad .50$

HEDGING

E. Privet ..............\$ \$4.00 per 100

California Privet ..........\$5.00 per 100 

The following table is about the usual weight of different kinds of plants ready for shipment by Parcel Post.

Strawberries........100 plants, weight, $4 \mathrm{tbs}$. Raspberries ......... " " " 10 "

Blackberries ........ " " " 12 "

Dewberries ......... " " " " 5 "

Gooseberries ........." " " " $" 8$ "

Currants ..........." " " " 10 "

Asparagus .........." " " " 8 " "

Rhubarb .......... " " " 630 "

Grapes ............" " " 15 "

If you prefer, estimate the postage in your order and send enough to cover charges. If we receive more than is necessary to cover postage, we will refund balance to you or send extra plants to you if you wish.

COMPLAINTS, if any, must be made within ten days after stock is received. We will then investigate quickly and carefully, making things right at once if the fault is ours. Please remember, we are never liable for more than cost of the plants.

\section{RASPBERRIES}

\begin{tabular}{|c|c|c|c|c|c|}
\hline & & Jach & 10 & 100 & 1000 \\
\hline Cuthbert & $\ldots \$$ & .05 & $\$ .40$ & $\$ 1.50$ & $\$ 10.00$ \\
\hline Antwerp & & .05 & .40 & 2.00 & 15.00 \\
\hline King . & & .10 & .50 & 3.00 & \\
\hline [arlboro & & .10 & .40 & 2.50 & \\
\hline egis & Everbearing. . & .10 & .75 & 5.00 & \\
\hline & & .15 & 1.00 & 7.50 & \\
\hline olden $\mathrm{Qu}$ & deen & .10 & .75 & 6.00 & \\
\hline
\end{tabular}

\section{BLACK CAPS}

Cumberland $\ldots \ldots \ldots \ldots . .10 \quad .75 \quad 7.00 \quad 45.00$

Plum Farmer ........ $.10 \quad .75 \quad 7.00 \quad 45.00$

Gregg $\quad \ldots \ldots \ldots \ldots \ldots \ldots .10 \quad .10 \quad .75 \quad 7.00 \quad 45.00$

Kansas $\ldots \ldots \ldots \ldots \ldots \ldots . .10 \quad .75 \quad 7.00 \quad 45.00$

Black Pearl ........... $.10 \quad .75 \quad 7.00 \quad 45.00$

Ohio ................ $.10 \quad .75 \quad 7.00 \quad 45.00$

\section{PURPLE RASPBERRIES}

Royal Purple ......... . .10 $\quad .75 \quad 7.00$

Columbian $\ldots \ldots \ldots \ldots . . .10 \quad .600$

45.00

Haymaker ............ 10

$.75 \quad 7.00$

Japanese Wine Berry.. $.15 \quad 1.00$

Strawberry Raspberry.. $\quad .15 \quad 1.00 \quad 7.50$

Phenomenal Berry .... $.30 \quad 2.50 \quad 17.50$

$\begin{array}{llllll}\text { Loganberry } \ldots \ldots \ldots \ldots & .20 & 1.25 & 10.00 & 75.00\end{array}$

BLACKBERRIES

$\begin{array}{llllll}\text { Rathbun } \ldots \ldots \ldots \ldots \ldots & .10 & .60 & 5.00 & 50.00 \\ \text { Lawton } \ldots \ldots \ldots \ldots \ldots \ldots & .10 & .60 & 5.00 & 30.00 \\ \text { Kittatinny } \ldots \ldots \ldots \ldots \ldots & .10 & .60 & 5.00 & 30.00 \\ \text { Early King } \ldots \ldots \ldots \ldots \ldots & .10 & .60 & 5.00 & 30.00\end{array}$


BLACK BERRIES-(Continued)

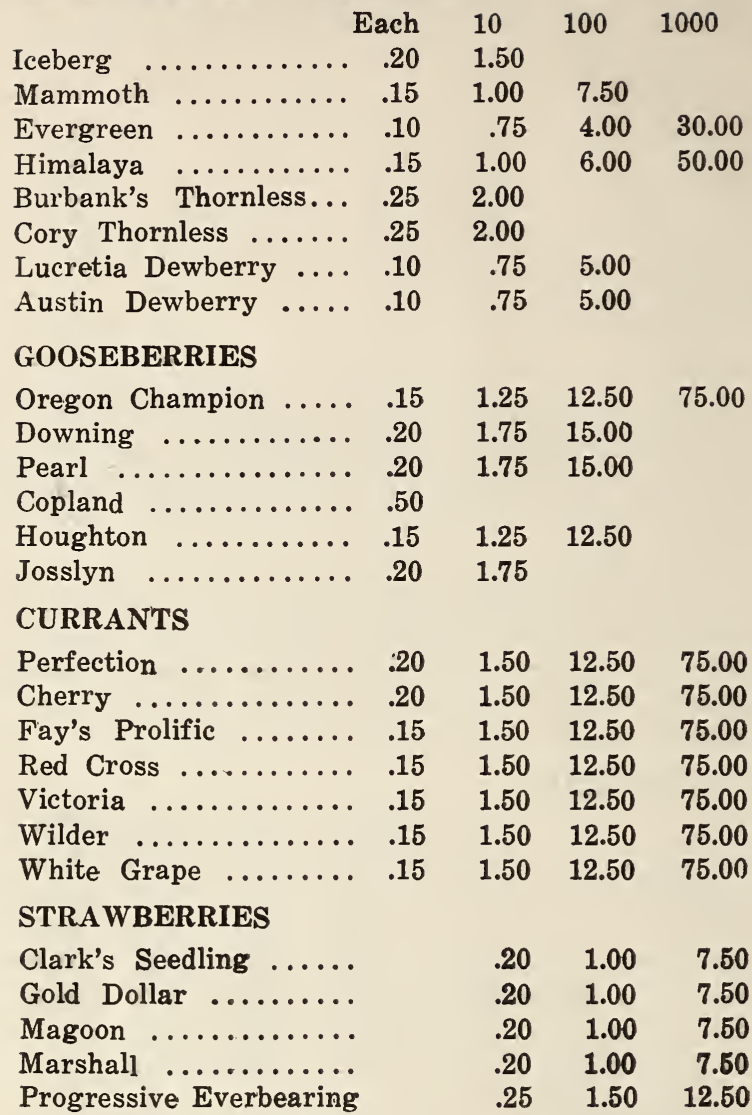

GRAPE VINES

Moore's Early ....... .25

$2.50 \quad 15.00$

Concord .............25

$2.50 \quad 15.00$

Agawam ............25

$2.50 \quad 15.00$

Brighton ........... .25

$2.50 \quad 15.00$

Niagara ..............25

$2.50 \quad 15.00$

Moore's Diamond ..... .25

$2.50 \quad 15.00$

Island Belle ........ .35

\section{RHUBARB}

$\begin{array}{llllll}\text { Mammoth Victoria } \ldots . . . & .15 & 1.00 & 6.00 & 50.00\end{array}$

Burbank Giant Winter $.20 \quad 1.50$

HORSERADISH

HOP ROOTS

ASPARAGUS

Palmetto ............

Conover's Colossal

Mammoth White ...... 